\title{
Downregulation of miR-19a exhibits inhibitory effects on metastatic renal cell carcinoma by targeting PIK3CA and inactivating Notch signaling in vitro
}

\author{
WEI XIAO, ZHIYONG GAO, YIXING DUAN, WUXIONG YUAN and YANG KE \\ Department of Urology, Hunan Provincial People's Hospital, Changsha, Hunan 410005, P.R. China
}

Received January 21, 2015; Accepted April 28, 2015

DOI: $10.3892 /$ or.2015.4041

\begin{abstract}
Metastasis seriously affects the clinical outcome of renal cell carcinoma patients. Despite the approval of several targeted therapies that have led to an improvement in the progression-free survival rate for these patients, advanced and metastatic renal cell carcinoma remains difficult to treat. Recently, microRNAs have been found to play a critical role in the metastatic dissemination of renal cell carcinoma cells. In the present study, we found that miR-19a expression was significantly upregulated in metastatic clear cell renal carcinoma than that in adjacent and primary carcinoma tissues using qPCR and in situ hybridization experiments. In addition, the results were confirmed in renal carcinoma cell lines. miR-19a expression in the cell lines derived from a metastatic site was higher than that in cell lines derived from a primary site. By gain- and loss-of-function experiments, we found that miR-19a acted as an oncogenic miRNA regulating renal cancer cell proliferation, migration and invasion by directly targeting PIK3CA. Furthermore, we also explored the downstream molecules of miR-19a/PIK3CA signaling. We found that Notch signaling was induced by upregulation of miR-19a, and inactivation of Notch signaling attenuated the cell proliferation, migration and invasion promoted by miR-19a. Thus, we provide evidence demonstrating that downregulation of miR-19a may be therapeutically beneficial for metastatic renal carcinoma.
\end{abstract}

\section{Introduction}

Renal cell carcinoma ( $\mathrm{RCC}$ ) is the most common carcinoma of the adult kidney and accounts for $\sim 3 \%$ of all cancer cases in adults with the highest mortality rate of over $40 \%$ (1). Clear cell RCC (ccRCC) is the major subtype of RCC accounting for more than $80 \%$ of all RCC types (2). If ccRCC is limited

Correspondence to: Dr Wei Xiao, Department of Urology, Hunan Provincial People's Hospital, Jiefang West Road 61, Changsha, Hunan 410005, P.R. China

E-mail: hphxiaowei@163.com

Key words: renal cell carcinoma, miR-19a, metastasis, PIK3CA, Notch signaling to the kidneys and has no systemic metastatic spread, it is a potentially curable disease, particularly when diagnosed at an early stage (3). Metastasis significantly affects the clinical outcome of RCC patients. Despite the approval of several targeted therapies that have led to an improvement in the progression-free survival rate of these patients, advanced and metastatic RCC remains difficult to treat (4). Understanding the molecular mechanisms involved in metastatic RCC may improve the outcome of RCC patients. Thus, more efforts are urgently needed to demonstrate the potential mechanisms underlying the metastasis of RCC. Recently, microRNAs, a class of small non-coding RNAs, were found to play critical roles in the metastatic dissemination of tumor cells in different types of cancer, including ccRCC (5).

miR-19a has been identified as an oncogenic miRNA in gastric (6) and bladder cancer (7) and glioma (8). In addition, miR-19a appears to be a tumor suppressor in some types of cancer, such as multiple myeloma (9). Thus, miR-19a is a bifunctional miRNA depending on various cancer types. However, the role of miR-19a in renal cancer remains unknown.

It has been reported that modulation of the miR-19/PTEN/ AKT/p53 axis by curcumin inhibited bisphenol A-associated breast cancer progression (10). The PIK3/AKT signaling pathway has been implicated in the metastasis of urothelial carcinoma, such as renal carcinoma (11). Phosphoinositide-3kinase catalytic $\alpha$ subunit (PIK3CA) and PTEN, integral parts of this pathway, play a critical role in controlling malignant growth, cell cycle progression and proliferation (11). The mutations identified in PIK3CA and PTEN are present in renal metastasis of adenoid cystic carcinoma of the breast, coinciding with a decrease in their expression levels, suggesting that inactivation of the PI3K/AKT pathway may be responsible for the unusually aggressive course of adenoid cystic carcinoma (12). Thus, we hypothesized that miR-19a/PIK3CA signaling may play a role in metastatic renal carcinoma.

In the present study, we found that miR-19a expression was significantly upregulated in metastatic ccRCC when compared with that in adjacent and primary carcinoma tissues using qPCR and in situ hybridization experiments. In addition, these results were confirmed in renal carcinoma cell lines. miR-19a expression in the cell lines derived from a metastatic site was higher than that of cell lines derived from a primary site. By gain- and loss-of-function experiments, we found that miR-19a 
acted as an oncogenic miRNA regulating renal cancer cell proliferation, migration and invasion by directly targeting PIK3CA. Furthermore, we also explored the downstream molecules of miR-19a/PIK3CA signaling. Recent studies have indicated that the Notch signaling pathway plays an important role in renal function (13). A previous study demonstrated that high expression of Notch signaling molecules, Notch1 and its ligand Jagged1, increased the risk of metastasis in T1 stage ccRCC (14). In the present study, we found that Notch signaling was induced by upregulation of miR-19a, and inactivation of Notch signaling attenuated cell proliferation, migration and invasion promoted by miR-19a. Thus, we provide evidence to demonstrate that downregulation of miR-19a may be therapeutically beneficial for metastatic renal carcinoma.

\section{Materials and methods}

Sample collection. A total of 60 clear cell renal cell carcinoma tissue samples, including 20 adjacent, 20 primary and 20 metastatic tumor tissues were obtained from the Hunan Provincial People's Hospital according to the Legislation and Ethics Boards of Hunan Provincial People's Hospital. Informed consent was obtained by all subjects. All samples were collected and classified using histopathological evaluation and stored at $-80^{\circ} \mathrm{C}$ until used.

Cell culture and treatment. Human renal tubular epithelial cells (HKC) and all human renal carcinoma cell lines, including ACHN, Caki-1, 786-O, SN12C, A704, A498 and TK10, were purchased from the American Type Culture Collection (ATCC; Manassas, VA, USA). All cells were cultured in RPMI-1640 medium (Life Technologies, Grand Island, NY, USA) supplemented with $10 \%$ fetal bovine serum (FBS) under standard conditions $\left(5 \% \mathrm{CO}_{2}, 37^{\circ} \mathrm{C}\right)$. The cells were transfected with lentiviruses that expressed pre-miR-19a, anti-miR-19a or negative control (NC) using Lipofectamine 3000 (Invitrogen, Carlsbad, CA, USA) at a final concentration of $50 \mathrm{nM}$. The lentiviruses for knockdown of Notch1 and Jagged1 were designed and purchased from Genechem (Shanghai, China). Following a 48-h transfection, the expression of miR-19a was detected by real-time PCR and the expression levels of PIK3CA, Notch1, Jagged1 and MMP9 were assessed by western blot analysis.

In situ hybridization for miR-19a. The paraffin-embedded tissue samples were serially cut at $4 \mu \mathrm{m}$. Slides were deparaffinized in dimethylbenzene and dehydrated in gradient alcohol. Expression of miR-19a was detected by in situ hybridization with probes for miR-19a according to the manufacturer's protocol provided in the microRNA ISH Optimization kit (Exiqon Inc., Vedbaek, Denmark). The probe sequence was designed as UGUGCAAAUCUAUGCAAAACUGA. The slides were visualized with 3,3'-diaminobenzidine (DAB) for $5 \mathrm{~min}$, and counterstained with haematoxylin for $1 \mathrm{~min}$. Results of the slides were evaluated, and images were captured with an Olympus microscope (Olympus C-7070; Tokyo, Japan).

Quantitative real-time polymerase chain reaction ( $q P C R)$. Total RNA was extracted from the indicated cells using the Ultrapure RNA kit (CWBiotech, Beijing, China) according to the manufacturer's instructions. The expression of miR-19a was detected by qPCR using miRcute miRNA (Qiagen, Valencia, CA, USA). The specific primer sets for miRNA-19a (HmiR0201) and U6 (miRQP9001) were purchased from GeneCopoeia. miR-19a expression was normalized to U6. The expression of PIK3CA was detected by qPCR using the FastLane Cell SYBR ${ }^{\circledR}$ Green kit (Qiagen). The primers for PIK3CA and $\beta$-actin were: PIK3CA sense, TGCTAAAGA GGAACACTGTCCA and antisense, GGTACTGGCCAAA GATTCAAAG; $\beta$-actin sense, AGGGGCCGGACTCGT CATACT and antisense, GGCGGCACCACCATGTACCCT. The $2^{-\Delta \Delta C T}$ method was used to analyze the data, and $\beta$-actin expression was used as internal control.

Western blot analysis. The total protein was extracted from the indicated cells using RIPA lysis buffer (Boster, Wuhan, China). Protein concentrations were determined using the BCA protein assay kit (Thermo, Waltham, MA, USA). A total of $60 \mu \mathrm{g}$ total protein was separated using SDS-PAGE and then transferred to nitrocellulose membranes. The membranes were blocked in $8 \%$ non-fat milk diluted in TBST and incubated with the indicated primary antibody [polyclonal antibody anti-PIK3CA and anti-MMP9 (from ABZOOM; rabbit, 1:200); polyclonal antibody anti-Notch1 and anti-Jagged1 (from Immunoway; rabbit, 1:100); monoclonal antibody anti- $\beta$-actin (from Boster; mouse, $1: 3,000$ )] overnight at $4^{\circ} \mathrm{C}$. The membranes were washed with TBST for three times for 15 min each and incubated with the secondary antibody for $60 \mathrm{~min}$ at $37^{\circ} \mathrm{C}$. The signals on the membrane were detected by enhanced chemiluminescence reagent. $\beta$-actin was used as as internal control expression. Data were analyzed by densitometry using Image-Pro plus software 6.0.

Dual luciferase report system. The wild-type (wt) and mutant (mut) 3'-untranscriptional region (3'-UTR) of PIK3CA were designed by GeneCopoeia, and were inserted into the dual luciferase reporter vector. For the luciferase assay, $10^{5}$ cells were plated and cultured in 12-well plates to reach $\sim 70 \%$ confluency. 786-O and Caki-1 cells were co-transfected with miR-19a mimic or miR-19a inhibitors and wt/mut 3'-UTR of PIK3CA dual luciferase reporter vector, respectively. Following a 48-h transfection, the luciferase activities were detected using a dual luciferase reporter gene assay kit (BioVision, Milpitas, CA, USA) on a luminometer (Roche, Mannheim, Germany). Renilla luciferase activity was normalized to firefly luciferase activity.

Transwell assay. Starved cells were resuspended in serumfree medium and added to the upper chamber of a Transwell chamber. The lower chamber was filled with medium containing $10 \%$ FBS. Following a 24-h culture, the cells that attached to the bottom were fixed and stained with crystal violet for $45 \mathrm{~min}$ and dried in air. The optical density (OD) at $570 \mathrm{~nm}$ of the crystal violet dissolved in $10 \%$ acetic acid was detected using an enzyme immunoassay analyzer.

CCK-8 assay. Five thousand cells were seeded in each 96-well plate. Then, following the indicated treatment, the cells were further incubated for $0,24,48$ and $72 \mathrm{~h}$, respectively. Ten microliters of CCK- 8 reagents (Solarbio, Beijing, China) were 

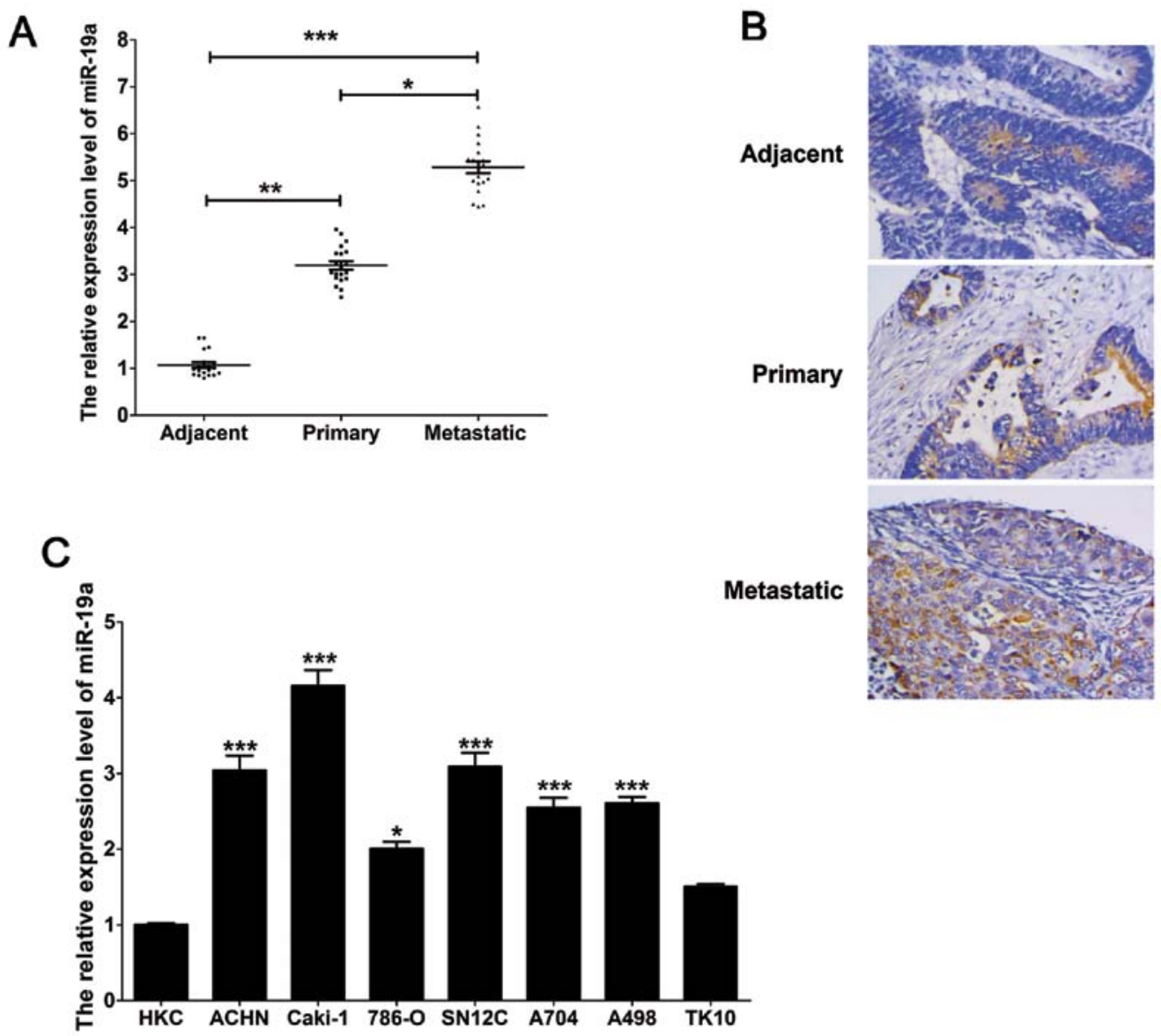

Figure 1. miR-19a expression in renal carcinoma tissues and cell lines. (A) miR-19a expression levels were significantly upregulated in primary and metastatic clear cell renal carcinoma tissues compared with that of adjacent tissues. (B) Representative images of in situ hybridization for miR-19a in adjacent, primary and metastatic clear cell renal carcinoma tissues. Magnification, x200. (C) miR-19a expression levels were significantly upregulated in renal carcinoma cell lines compared with that in the HKC control cells. Data are presented as means $\pm \mathrm{SD} .{ }^{*} \mathrm{P}<0.05,{ }^{* *} \mathrm{P}<0.01$ and ${ }^{* * *} \mathrm{P}<0.001$.

added to the well at $1 \mathrm{~h}$ before the end of the incubation. The OD at $570 \mathrm{~nm}$ of each well was detected by enzyme immunoassay analyzer.

Scratch assay. Cells in each group were collected and resuspended in complete medium containing $10 \%$ FBS. Then, $5 \times 10^{4}$ cells were seeded in 24 -well plates and cultured at $37^{\circ} \mathrm{C}$ in $5 \% \mathrm{CO}_{2}$ until reaching $\sim 100 \%$ confluency. The cells were scratched with the head of a 10- $\mu$ l tip and the cells were gently washed with serum-free medium. Following a 24-h culture in serum-free medium at $37^{\circ} \mathrm{C}$ in $5 \% \mathrm{CO}_{2}$, the cells were cultured in complete medium containing $10 \%$ FBS for three days. Cells in each group were photographed.

Statistical analysis. Data analysis was performed using Graphpad Prism5 software and SPSS 16.0 software. Depending on the experimental conditions, Student's t-tests or one-way ANOVA were used. Data are expressed as mean \pm SD. P-values $<0.05$ were considered statistically significant compared to the controls.

\section{Results}

miR-19a is upregulated in renal carcinoma tissues and cell lines. The average expression level of miR-19a was significantly upregulated $(\mathrm{P}<0.001)$ in the renal carcinoma tissue samples, including primary and metastatic clear cell renal carcinoma tissues, compared with the 20 adjacent controls, as indicated by qPCR. In addition, the expression of miR-19a in the metastatic clear cell renal carcinoma tissues was higher than that in the primary tissues (Fig. 1A). Furthermore, these results were confirmed by in situ hybridization (Fig. 1B). Moreover, similar results were observed in the renal carcinoma cell lines. The expression of miR-19a in the renal carcinoma cell lines was significantly higher than that in the HKC cells. Moreover, miR-19a expression in the ACHN and Caki-1 cells derived from a metastatic site was higher than that in cells derived from a primary clear cell adenocarcinoma, including 786-O, SN12C, A704, A498 and TK10 cells (Fig. 1C).

miR-19a regulates PIK3CA expression at the transcriptional and translational levels by directly targeting its 3'-UTR. To further investigate the downstream molecules targeted by miR-19a, we transfected Lv-pre-miR-19a or Lv-anti-miR-19a into 786-O and Caki-1 cells to induce or knock down the expression of miR-19a. We found that miR-19a expression was markedly upregulated or downregulated in the 786-O and Caki-1 cells following Lv-pre-miR-19a or Lv-anti-miR-19a transfection, indicating that the efficiency of transfection was satisfied for further analysis (Fig. 2A and D). Furthermore, 
A

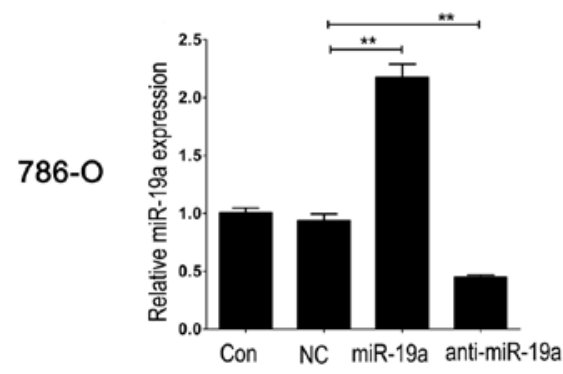

B

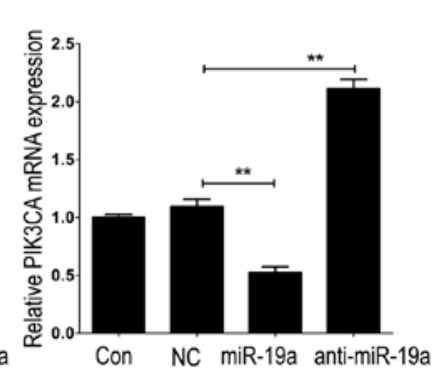

E

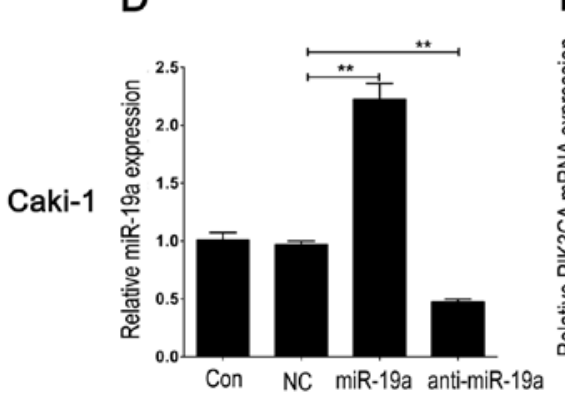

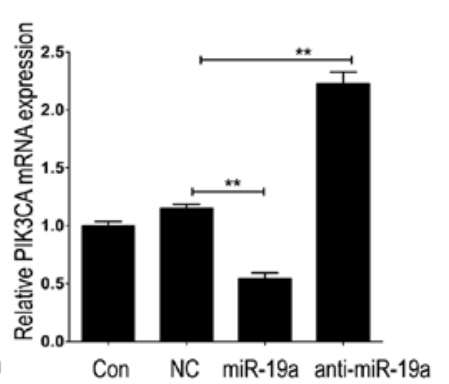

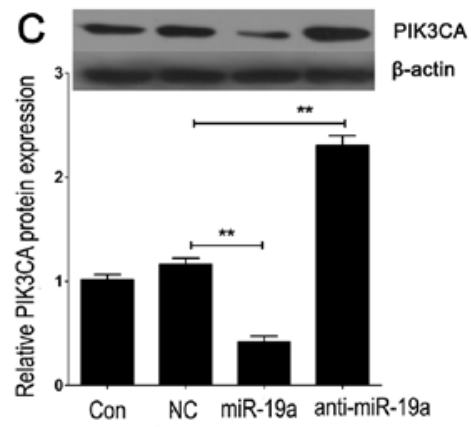

F $--\infty$ PIK3CA

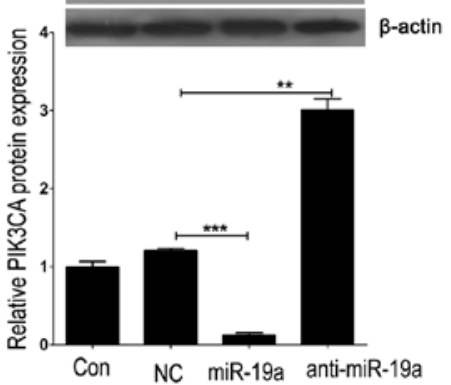

Figure 2. miR-19a regulates PIK3CA expression at the transcriptional and translational levels. (A and D) Following Lv-pre-miR-19a or Lv-anti-miR-19a transfection, qPCR was used to detect the expression of miR-19a in the 786-O (A) and Caki-1(B) cells. (B and E) Following Lv-pre-miR-19a or Lv-anti-miR-19a transfection, qPCR was used to detect the mRNA expression of PIK3CA in the 786-O (B) and Caki-1 (E) cells. (C and F) Following Lv-pre-miR-19a or Lv-antimiR-19a transfection, western blot analysis was used to detect the expression of PIK3CA protein in the 786-O (C) and Caki-1 (F) cells. Data are presented as means \pm SD. ${ }^{*} \mathrm{P}<0.05,{ }^{* *} \mathrm{P}<0.01$ and ${ }^{* * * *} \mathrm{P}<0.001$.
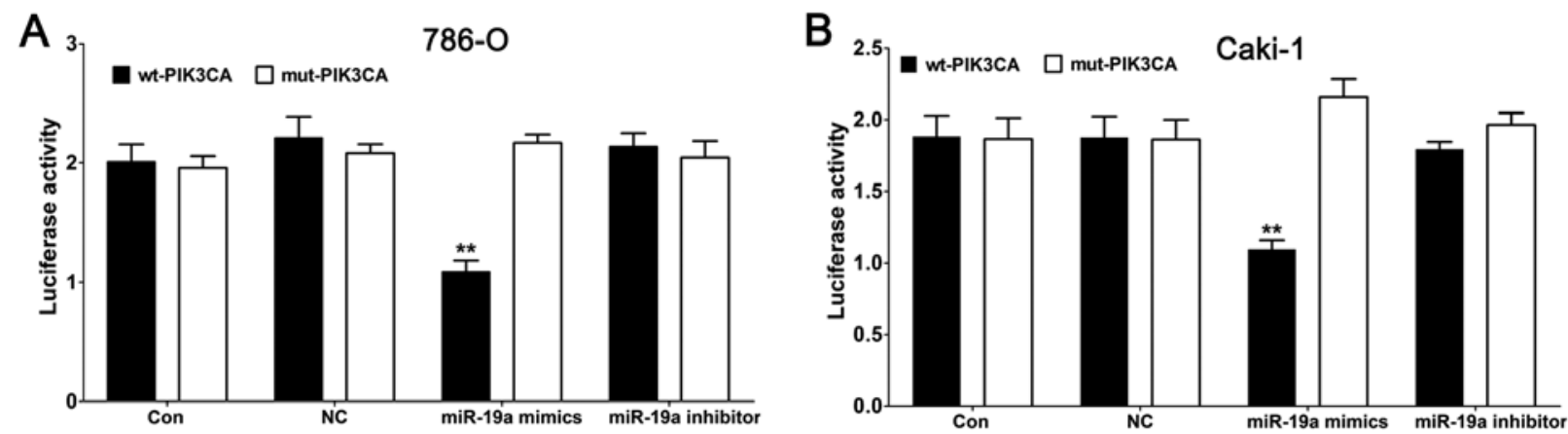

Figure 3. miR-19a directly targets PIK3CA 3'-UTR. (A and B) Lv-pre-miR-19a or Lv-anti-miR-19a was co-transfected with wt-PIK3CA or mut-PIK3CA into 786-O or Caki-1 cells, respectively. Compared to the NC, the luciferase activity of wt-PIK3CA was decreased significantly by pre-miR-19a, which was abrogated by mut-PIK3CA. The luciferase activity had no significant changes in the 786-O or Caki-1 cells treated with anti-miR-19a. Data are presented as means $\pm \mathrm{SD}$. ${ }^{*} \mathrm{P}<0.05$ and ${ }^{* *} \mathrm{P}<0.01$.

mRNA and protein expression levels of PIK3CA, a putative target of miR-19a screened by a bioinformatic tool (Targetscan), were markedly downregulated by Lv-pre-miR-19a transfection and upregulated by Lv-anti-miR-19a transfection, compared with the control in the 86-O and Caki-1 cell lines (Fig. 2B, $\mathrm{C}, \mathrm{E}$ and $\mathrm{F})$. We then aimed to ascertain whether the 3'-UTR of PIK3CA had a direct target site for miR-19a. Vectors encoding the wt or mut 3'-UTR of PIK3CA were constructed into a dual luciferase reporter gene. By dual luciferase reporter assay, we found that the luciferase activity was significantly repressed in the miR-19a mimic transfectant compared to the NC transfectant. Moreover, miR-19a-mediated repression of luciferase activity was abolished by the mutant-type 3'-UTR of PIK3CA (Fig. 3A and B). These results demonstrated that miR-19a directly targets PIK3CA and regulates its expression at the transcriptional and translational levels.

Effects of miR-19a on cell proliferation, migration and invasion in 786-O and Caki-1 cells. 786-O and Caki-1 cell proliferation was measured using CCK- 8 assay following overexpression or knockdown of miR-19a. We found that overexpression of miR-19a induced promotion of proliferation, while downregulation of miR-19a inhibited cell proliferation in the 786-O and Caki-1 cell lines (Fig. 4A). In addition, a scratch assay was used to analyze cell migration after overexpression or knockdown of miR-19a. Upregulation of miR-19a 
A

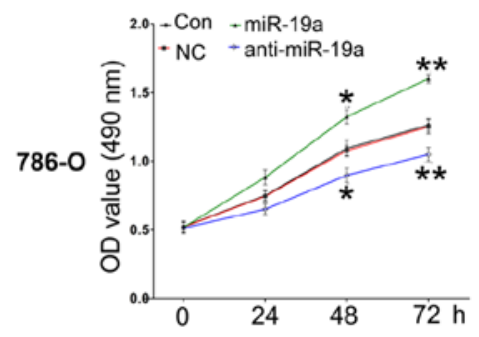

B

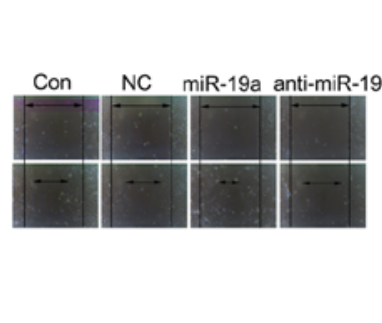

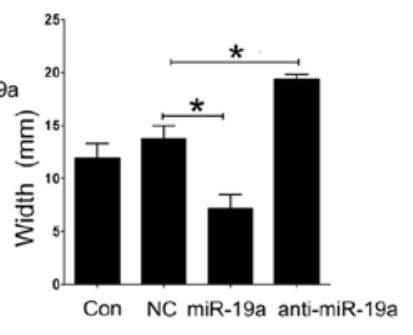

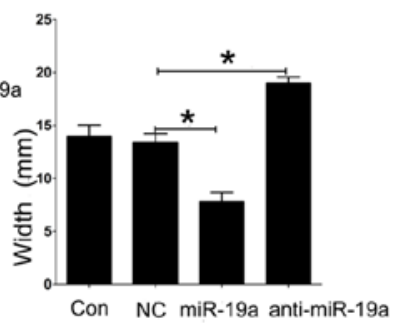

C

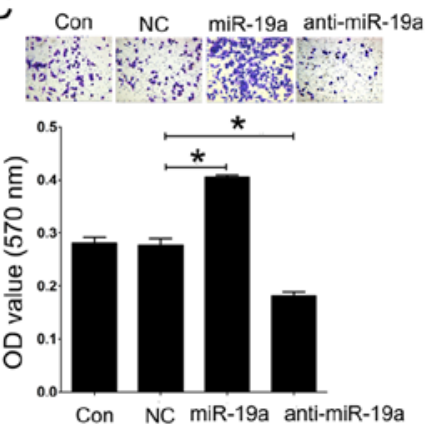

Con NC miR-19a anti-miR-19a

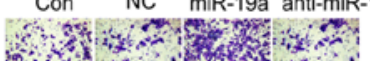

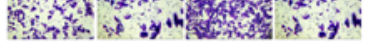

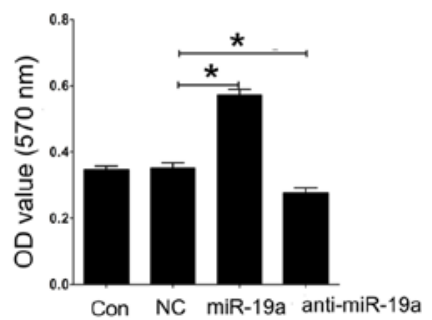

Figure 4. Effect of miR-19a on cell proliferation, migration and invasion. (A) Following Lv-pre-miR-19a or Lv-anti-miR-19a transfection, CCK-8 assay was used to measured cell proliferation of the 786-O (upper panel) and Caki-1 (lower panel) cells. (B) Following Lv-pre-miR-19a or Lv-anti-miR-19a transfection, scratch assay was used to analyze cell migration of 786-O (upper panel) and Caki-1 (lower panel) cells. (C) Following Lv-pre-miR-19a or Lv-anti-miR-19a transfection, Transwell assay was used to analyze cell invasion of the 786-O (upper panel) and Caki-1 (lower panel) cells. Data are presented as means \pm SD. ${ }^{*} \mathrm{P}<0.05,{ }^{* *} \mathrm{P}<0.01$ and ${ }^{* * *} \mathrm{P}<0.001$.
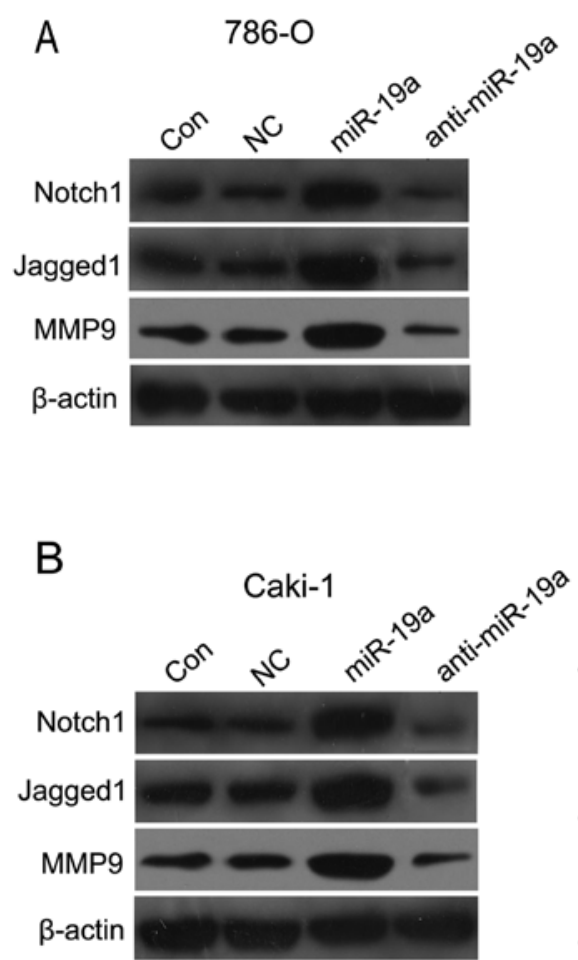
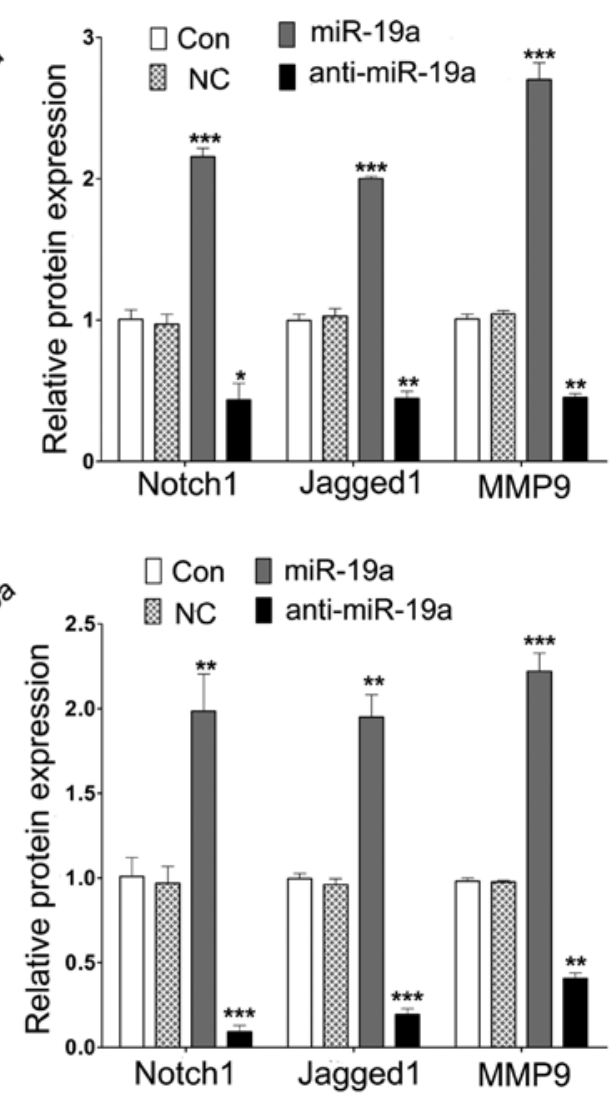

Figure 5. Effect of miR-19a on the expression of Notch1, Jagged1 and MMP9. (A) Following Lv-pre-miR-19a or Lv-anti-miR-19a transfection, western blot analysis was used to detect the expression of Notch1, Jagged1 and MMP9 protein in the 786-O cells. (B) Following Lv-pre-miR-19a or Lv-anti-miR-19a transfection, western blot analysis was used to detect the expression of Notch1, Jagged1 and MMP9 protein in the Caki-1 cells. Data are presented as means $\pm \mathrm{SD} .{ }^{*} \mathrm{P}<0.05,{ }^{* * *} \mathrm{P}<0.01$ and $^{* * * *} \mathrm{P}<0.001$. 
A

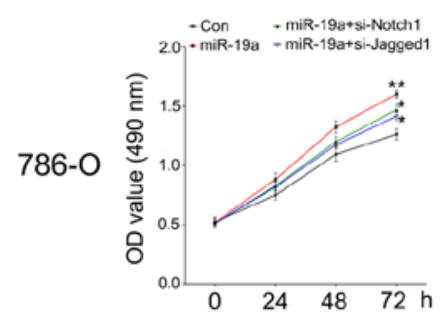

B
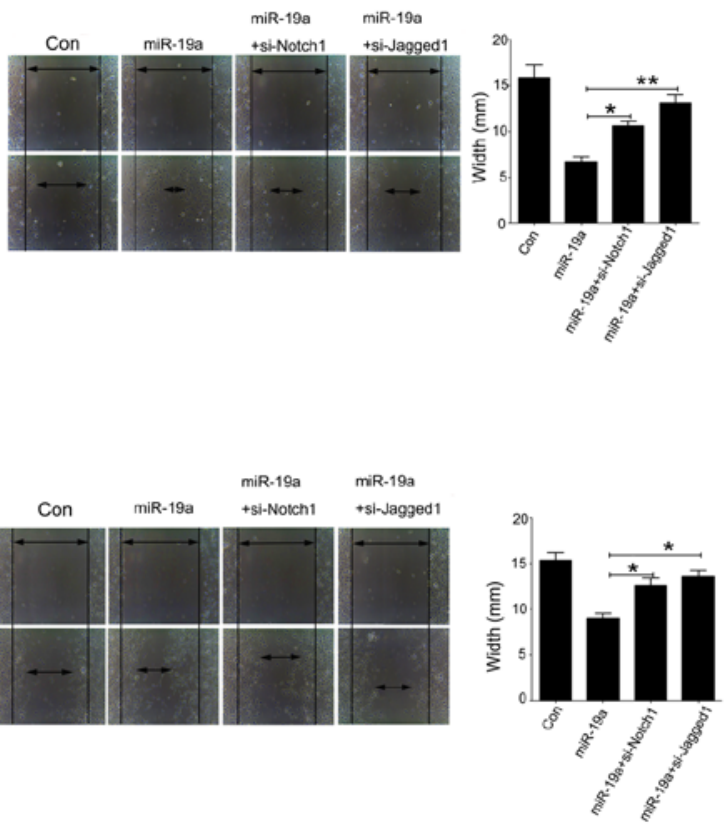

C
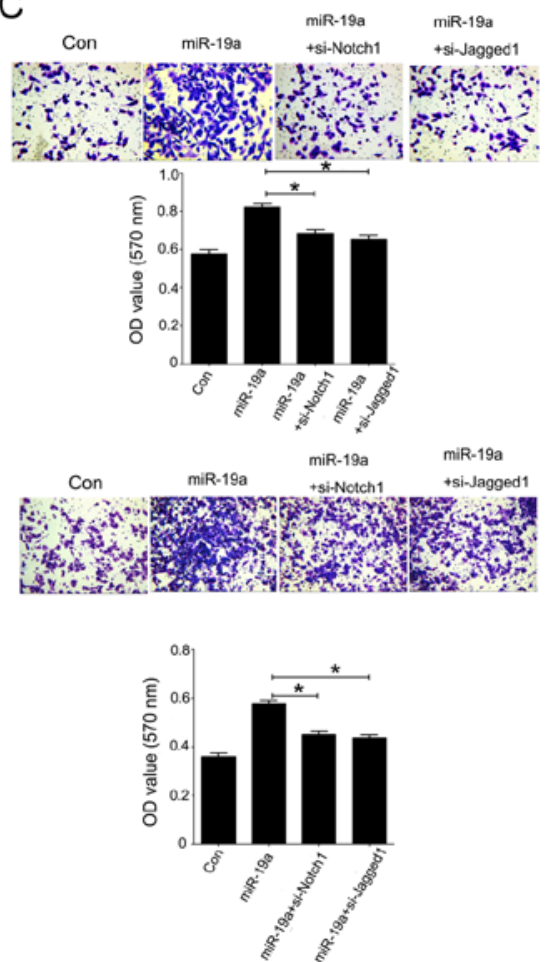

Figure 6. Knockdown of Notch1 or Jagged1 regulates cell proliferation, migration and invasion. (A) Following siRNA-Notch1 or siRNA-Jagged1 co-transfected with Lv-pre-miR-19a, CCK-8 assay was used to measured cell proliferation of the 786-O (upper panel) and Caki-1 (lower panel) cells. (B) Following siRNA-Notch1 or siRNA-Jagged1 co-transfected with Lv-pre-miR-19a, scratch assay was used to measured cell migration of the 786-O (upper panel) and Caki-1 (lower panel) cells. (C) Following siRNA-Notch1 or siRNA-Jagged1 co-transfected with Lv-pre-miR-19a, Transwell assay was used to measured cell invasion of the 786-O (upper panel) and Caki-1 (lower panel) cells. Data are presented as means \pm SD. ${ }^{*} \mathrm{P}<0.05,{ }^{* * *} \mathrm{P}<0.01$ and ${ }^{* * *} \mathrm{P}<0.001$.

significantly induced cell migration compared to $\mathrm{NC}$, while knockdown of miR-19a repressed cell migration in the 786-O and Caki-1 cell lines (Fig. 4B). Furthermore, using a Transwell assay, we found that introduction of miR-19a expression significantly induced cell invasion compared to NC, while knockdown of miR-19a had an opposite effect on the 786-O and Caki-1 cell lines (Fig. 4C).

miR-19a regulates Notch1, Jagged1 and MMP9 expression. To explore potential downstream molecular pathways underlying miR-19a targeting to PIK3CA, we assessed the expression of proliferation- and invasion-related genes including Notch1, Jagged1 and MMP9 by western blot analysis in the 786-O and Caki-1 cells following Lv-pre-miR-19a or Lv-anti-miR-19a transfection. A significant increase in the expression of Notch1, Jagged1 and MMP9 proteins was observed in the cells treated with Lv-pre-miR-19a. Inversely, knockdown of miR-19a significantly reduced the expression of Notch1, Jagged1 and MMP9 proteins in the 786-O and Caki-1 cells compared with that in the control (Fig. 5A and B).

Notchl and Jagged1 siRNAs attenuate the effects of miR-19a on cell proliferation, migration and invasion in 786-O and Caki-1 cells. To investigate the role of Notch1 and Jagged1 signaling in the miR-19a/PIK3CA pathway in renal carcinoma cells, a loss-of-function experiment was performed. As shown in Fig. 6A, CCK-8 assay was used to determine the proliferation of the 786-O and Caki-1 cells following overex- pression of miR-19a and transfection with siRNA-Notch1 or siRNA-Jagged1. Knockdown of Notch1 or Jagged1 inhibited the cell proliferation promoted by miR-19a in the 786-O and Caki-1 cells. In addition, a scratch assay was used to analyze cell migration after knockdown of Notch1 or Jagged1 in the cells treated with Lv-pre-miR-19a. Downregulation of Notch1 or Jagged1 significantly suppressed cell migration compared to the Lv-pre-miR-19a group in the 786-O and Caki-1 cell lines (Fig. 6B). Furthermore, using a Transwell assay, we found that knockdown of Notch1 or Jagged1 significantly attenuated cell invasion induced by miR-19a in the 786-O and Caki-1 cell lines (Fig. 6C).

\section{Discussion}

Due to metastasis, ccRCC, the most common histologic subtype of RCC, has become the leading cause of death from adult urologic tumors. Emerging evidence indicates that miRNAs may act as either oncogenes or tumor suppressors and participate in the metastasis of malignancies. It has been reported that upregulation of miR-19a has an oncogenic role in gastric cancer (6) and in supraglottic carcinoma tissues (15), and high expression of miR-19a was significantly associated with a poor patient survival rate in human astrocytoma (16) and correlates with more aggressive phenotypes of bladder cancer (7). In addition, overexpression of miR-19a was noted in early-stage breast cancer patients and the level was significantly decreased after chemo/radiotherapy; in the case 
of high-risk breast cancer patients serum levels of miR-19a were significantly more frequent in comparison to a low-risk group (17). Consistent with a previous study, we found that miR-19a was significantly increased in ccRCC tissues and renal carcinoma cell lines, with a higher expression in metastatic than in localized tissues, suggesting that miR-19a is involved in renal carcinoma progression. Furthermore, by gain- and loss-of-function experiments, we found that enforced miR-19a expression facilitated renal cancer cell proliferation, migration and invasion, whereas knockdown of miR-19a expression exhibited an opposite effect in vitro. Thus, collectively, upregulation of miR-19a in renal cancer may promote cancer cell metastasis and the poor outcome of patients.

Using a bioinformatic tool, we screened the putative targets of miR-19a and found that miR-19a conversely targets PIK3CA, an indispensable molecule of the mammalian target of rapamycin mTOR signaling and $\mathrm{PI} 3 \mathrm{~K} / \mathrm{AKT}$ pathway, which is deregulated in most types of cancer (18). PIK3CA mutations have been associated with a worse survival rate in metastatic colorectal (19) and breast cancer (20). Using a dual luciferase reporter gene system, we confirmed the results from the bioinformatic tool, that miR-19a regulates PIK3CA expression at the translational and transcriptional levels by targeting its 3'-UTR. It was previously demonstrated that overexpression of miR-19a was significantly associated with the metastasis of gastric cancer and poor overall prognosis at the clinical tissue level and promoted epithelial-mesenchymal transition through the PI3K/AKT pathway, and silencing of the PI3K/AKT pathway abolished the effect of miR-19a (6). PTEN, a critical molecule of the PI3K/AKT pathway, was found to be a target of miR-19b in prostate epithelial and cancer cells. Expression of miR-19b altered the expression of key components in the PI3K/Akt pathway, including PIK3CA resulting in acceleration of epithelial and prostate cancer cell proliferation (21). However, it was reported that a majority of non-small cell lung cancer tissue samples showed low miR-1 expression and high PIK3CA expression; miR-1 upregulation inhibited non-small cell lung cancer cell proliferation, migration and invasion by directly targeting PIK3CA (22). Wang et al demonstrated that siRNA-mediated silencing of PIK3CA blocked the inhibitory effect of miR-375 on colorectal cancer cell growth (23). Thus, PIK3CA exhibited an opposite effect depending on which miRNA was its target and on tumor type. In the present study, we found that PIK3CA was targeting by miR-19a and its upregulation was accompanied by inhibition of renal carcinoma cell growth and invasion.

Furthermore, we next explored the downstream molecules associated with the miR-19a/PIK3CA pathway. Suppression of the PI3K/Akt signaling pathway was associated with the Notch/Jagged pathway in several types of cancer (24). We found that induced expression of miR-19a by lentivirus resulted in a significant increase in Notch signaling and MMP9 level in renal cancer cell lines while downregulation of miR-19a decreased the expression levels of these genes. It was reported that high MMP9 expression levels are associated with poor prognosis of RCC (25). Furthermore, a poor survival rate with a high frequency of metastases in conventional RCC was found to be associated with MMP9 (26). Blockade of MMP9 inhibited the migration and invasion of RCC cells (27). In the present study, we showed that downregulation of miR-19a suppressed the MMP9 expression and subsequently suppressed migration and invasion of RCC cells. Notch genes, including Notch1, Notch2, Notch3 and Notch4, encode receptors for at least five different Notch ligands, including Jagged1. The Notch proteins mediate various cellular processes including differentiation, proliferation and apoptosis (28). It is possible that Notch pathway activation is a common mechanism in the pathophysiology of acquired renal diseases (29). Notch can act as either an oncogene or a tumor-suppressor gene in the development of cancer depending on the tumor type (30). A previous study showed that high expression of Notch1 and Jagged 1 increased the risk of metastasis in T1 stage ccRCC by stimulating the proliferation and migration of tumor cells (14). The expression of Notch1 and Jagged1 proteins was higher in RCC than in non-neoplastic tissues, correlative with tumor size, grade, TNM stage and disease relapse; and suppression of the Notch pathway was found to be associated with cell proliferation inhibition, as well as induced G2/M phase cell cycle arrest and cell apoptosis in 786-0 and Caki cell lines (31). Thus, the Notch pathway appears to be important in the oncogenesis of ccRCC. In the present study, we found that silencing of the Notch pathway by siRNA blocked the promotive effect of miR-19a on renal cancer cell growth, migration and invasion, suggesting that miR-19a contributes to the development of RCC through the Notch pathway via targeting PIK3CA.

In conclusion, high miR-19a expression was found to be associated with metastatic renal carcinoma tissues. Knockdown of miR-19a exhibited an inhibitory effect on renal cancer cell proliferation, migration and invasion by targeting PIK3CA, while siRNA-mediated silencing of Notch signaling attenuated the inhibitory effects of miR-19a. Thus, our evidence suggests that inactivation of miR-19a/Notch signaling may be therapeutically beneficial for the treatment and prevention of kidney cancer in the clinic.

\section{Acknowledgements}

The present study was supported by Hunan Provincial Science and Technology Program (2013FJ3149).

\section{References}

1. Dias F, Teixeira AL, Santos JI, Gomes M, Nogueira A, Assis J and Medeiros R: Renal cell carcinoma development and miRNAs: A possible link to the EGFR pathway. Pharmacogenomics 14: 1793-1803, 2013

2. Redova M, Svoboda M and Slaby O: MicroRNAs and their target gene networks in renal cell carcinoma. Biochem Biophys Res Commun 405: 153-156, 2011.

3. Mishra PJ: MicroRNAs as promising biomarkers in cancer diagnostics. Biomark Res 2: 19, 2014.

4. Hao JF, Ren KM, Bai JX, Wang SN, Shao B, Cao N and Li X: Identification of potential biomarkers for clear cell renal cell carcinoma based on microRNA-mRNA pathway relationships. J Cancer Res Ther 10 (Suppl): C167-C177, 2014.

5. Zhang L, Xul B, Chen S, Lu K, Liu C, Wang Y, Zhao Y, Zhang X, Liu D and Chen M: The complex roles of microRNAs in the metastasis of renal cell carcinoma. J Nanosci Nanotechnol 13: 3195-3203, 2013.

6. Lu W, Xu Z, Zhang M and Zuo Y: MiR-19a promotes epithelialmesenchymal transition through PI3K/AKT pathway in gastric cancer. Int J Clin Exp Pathol 7: 7286-7296, 2014.

7. Feng Y, Liu J, Kang Y, He Y, Liang B, Yang P and Yu Z: miR-19a acts as an oncogenic microRNA and is up-regulated in bladder cancer. J Exp Clin Cancer Res 33: 67, 2014. 
8. Jia Z, Wang K, Zhang A, Wang G, Kang C, Han L and Pu P miR-19a and miR-19b overexpression in gliomas. Pathol Oncol Res 19: 847-853, 2013.

9. Hao M, Zang M, Wendlandt E, Xu Y, An G, Gong D, Li F, Qi F, Zhang Y, Yang Y, et al: Low serum miR-19a expression as a nove poor prognostic indicator in multiple myeloma. Int J Cancer 136: $1835-1844,2014$

10. Li X, Xie W, Xie C, Huang C, Zhu J, Liang Z, Deng F, Zhu M, Zhu W, Wu R, et al: Curcumin modulates miR-19/PTEN/ $\mathrm{AKT} / \mathrm{p} 53$ axis to suppress bisphenol A-induced MCF-7 breast cancer cell proliferation. Phytother Res 28: 1553-1560, 2014.

11. Qian CN, Furge KA, Knol J, Huang D, Chen J, Dykema KJ, Kort EJ, Massie A, Khoo SK, Vanden Beldt K, et al: Activation of the PI3K/AKT pathway induces urothelial carcinoma of the renal pelvis: Identification in human tumors and confirmation in animal models. Cancer Res 69: 8256-8264, 2009.

12. Vranić S, Bilalović N, Lee LM, Kruslin B, Lilleberg SL and Gatalica Z: PIK3CA and PTEN mutations in adenoid cystic carcinoma of the breast metastatic to kidney. Hum Pathol 38: $1425-1431,2007$

13. Fujiki K, Inamura $\mathrm{H}$ and Matsuoka M: Detrimental effects of Notch1 signaling activated by cadmium in renal proximal tubular epithelial cells. Cell Death Dis 5: e1378, 2014.

14. Ai Q, Ma X, Huang Q, Liu S, Shi T, Zhang C, Zhu M, Zhang Y, Wang B, Ni D, et al: High-level expression of Notch1 increased the risk of metastasis in T1 stage clear cell renal cell carcinoma. PLoS One 7: e35022, 2012.

15. Zhang T, Han G, Wang Y, Chen K and Sun Y: MicroRNA expression profiles in supraglottic carcinoma. Oncol Rep 31: 2029-2034, 2014.

16. Zhi F, Shao N, Wang R, Deng D, Xue L, Wang Q, Zhang Y, Shi Y, Xia X, Wang S, et al: Identification of 9 serum microRNAs as potential noninvasive biomarkers of human astrocytoma. Neuro Oncol: Epub ahead of print: Aug 18, 2014. doi: 10.1093/neuonc/ nou169.

17. Sochor M, Basova P, Pesta M, Dusilkova N, Bartos J, Burda P, Pospisil V and Stopka T: Oncogenic microRNAs: miR-155, miR-19a, miR-181b, and miR-24 enable monitoring of early breast cancer in serum. BMC Cancer 14: 448, 2014.

18. Yu M,Bardia A, Aceto N, Bersani F, Madden MW, Donaldson MC, Desai R, Zhu H, Comaills V, Zheng Z, et al: Cancer therapy. Ex vivo culture of circulating breast tumor cells for individualized testing of drug susceptibility. Science 345: 216-220, 2014

19. Yaeger R, Cowell E, Chou JF, Gewirtz AN, Borsu L, Vakiani E, Solit DB, Rosen N, Capanu M, Ladanyi M, et al: RAS mutations affect pattern of metastatic spread and increase propensity for brain metastasis in colorectal cancer. Cancer 121: 1195-1203, 2014
20. Deb S, Wong SQ, Li J, Do H, Weiss J, Byrne D, Chakrabarti A, Bosma T, Fellowes A, Dobrovic A, et al; kConFab Investigators: Mutational profiling of familial male breast cancers reveals similarities with luminal A female breast cancer with rare TP53 mutations. Br J Cancer 111: 2351-2360, 2014.

21. Tian L, Fang YX, Xue JL and Chen JZ: Four microRNAs promote prostate cell proliferation with regulation of PTEN and its downstream signals in vitro. PLoS One 8: e75885, 2013.

22. Yu QQ, Wu H, Huang X, Shen H, Shu YQ, Zhang B, Xiang CC, Yu SM, Guo RH and Chen L: MiR-1 targets PIK3CA and inhibits tumorigenic properties of A549 cells. Biomed Pharmacother 68: 155-161, 2014.

23. Wang Y, Tang Q, Li M, Jiang S and Wang X: MicroRNA-375 inhibits colorectal cancer growth by targeting PIK3CA. Biochem Biophys Res Commun 444: 199-204, 2014.

24. Xiao W, Chen X and He M: Inhibition of the Jagged/Notch pathway inhibits retinoblastoma cell proliferation via suppressing the PI3K/Akt, Src, p38MAPK and Wnt/ $\beta$-catenin signaling pathways. Mol Med Rep 10: 453-458, 2014

25. Sato A, Nagase H, Obinata D, Fujiwara K, Fukuda N, Soma M, Yamaguchi K, Kawata N and Takahashi S: Inhibition of MMP-9 using a pyrrole-imidazole polyamide reduces cell invasion in renal cell carcinoma. Int J Oncol 43: 1441-1446, 2013.

26. Cho NH, Shim HS, Rha SY, Kang SH, Hong SH, Choi YD, Hong SJ and Cho SH: Increased expression of matrix metalloproteinase 9 correlates with poor prognostic variables in renal cell carcinoma. Eur Urol 44: 560-566, 2003.

27. Liang L, Li L, Zeng J, Gao Y, Chen YL, Wang ZQ, Wang XY, Chang LS and He D: Inhibitory effect of silibinin on EGFR signal-induced renal cell carcinoma progression via suppression of the EGFR/MMP-9 signaling pathway. Oncol Rep 28: 999-1005, 2012.

28. Li D, Masiero M, Banham AH and Harris AL: The notch ligand JAGGED1 as a target for anti-tumor therapy. Front Oncol 4: 254, 2014.

29. Murea M, Park JK, Sharma S, Kato H, Gruenwald A, Niranjan T, Si H, Thomas DB, Pullman JM, Melamed ML, et al: Expression of Notch pathway proteins correlates with albuminuria, glomerulosclerosis, and renal function. Kidney Int 78: 514-522, 2010.

30. Leong KG and Karsan A: Recent insights into the role of Notch signaling in tumorigenesis. Blood 107: 2223-2233, 2006.

31. Wu K, Zhang L, Lin Y, Yang K and Cheng Y: Inhibition of $\gamma$-secretase induces G2/M arrest and triggers apoptosis in renal cell carcinoma. Oncol Lett 8: 55-61, 2014. 